\title{
BMJ Open Effectiveness and safety of in vitro maturation of oocytes versus in vitro fertilisation in women with high antral follicle count: study protocol for a randomised controlled trial
}

\author{
Lan N Vuong, ${ }^{1,2}$ Vu N A Ho, ${ }^{2}$ Tuong $\mathrm{M} \mathrm{Ho},{ }^{2}$ Vinh Q Dang, ${ }^{2}$ Tuan H Phung, ${ }^{2}$ \\ Nhu H Giang, ${ }^{2}$ Anh H Le, ${ }^{2}$ Toan D Pham, ${ }^{2}$ Rui Wang, ${ }^{3}$ Rob J Norman,, 4 \\ Johan Smitz, ${ }^{5}$ Robert B Gilchrist, ${ }^{6}$ Ben W Mol ${ }^{7}$
}

To cite: Vuong LN, Ho VNA, Ho TM, et al. Effectiveness and safety of in vitro maturation of oocytes versus in vitro fertilisation in women with high antral follicle count: study protocol for a randomised controlled trial. BMJ Open 2018;8:e23413. doi:10.1136/ bmjopen-2018-023413

- Prepublication history and additional material for this paper are available online. To view these files, please visit the journal online (http://dx.doi org/10.1136/bmjopen-2018023413).

Received 5 April 2018 Revised 25 October 2018 Accepted 25 0ctober 2018

\section{Check for updates}

(c) Author(s) (or their employer(s)) 2018. Re-use permitted under CC BY-NC. No commercial re-use. See rights and permissions. Published by BMJ.

For numbered affiliations see end of article.

Correspondence to Dr Lan N Vuong; drlan@yahoo.com.vn; lanvuong@ump.edu.vn

\section{ABSTRACT}

Introduction In vitro maturation (IVM) is a potential alternative to conventional in vitro fertilisation (IVF) to avoid ovarian hyperstimulation syndrome (OHSS). This is particularly relevant in women with a high antral follicle count (AFC) and/or polycystic ovary syndrome (PCOS), who are at increased risk for OHSS. However, no randomised controlled trials of IVM versus IVF in women with high AFC have reported both pregnancy and OHSS rates. The aim of this study is to compare the effectiveness and safety of one IVM cycle and one IVF with segmentation cycle within women with PCOS or high AFC-related subfertility.

Methods and analysis This randomised controlled trial will be conducted at a specialist IVF centre in Vietnam. Eligible subfertile women with PCOS and/or high AFC will be randomised to undergo either IVM or IVF. The primary outcome is live birth after the first embryo transfer of the started treatment cycle. Cycles in which no embryo is available for transfer will be considered as failures. The study has a non-inferiority design, with a maximal acceptable between-group difference of $5 \%$. Rates of OHSS will also be reported.

Ethics and dissemination Ethical approval was obtained from the participating centre, and informed patient consent was obtained before study enrolment. Results of the study will be submitted for publication in a peer-reviewed journal.

Trial registration number NCT03405701; Pre-results.

\section{INTRODUCTION}

The prevalence of polycystic ovary syndrome (PCOS) ranges from about $5 \%$ to $20 \%$, and varies by ethnicity and the definition used. ${ }^{1}$ The proportion of subfertile women who have PCOS is about $50 \% .^{2}$ Although subfertile women with PCOS usually benefit from conventional treatments, such as ovulation induction and laparoscopic ovarian drilling, ${ }^{34}$ some will ultimately need assisted reproductive techniques such as controlled ovarian

\section{Strengths and limitations of this study}

The trial has a randomised, controlled design which should minimise bias

- There is a lack of data from randomised controlled trials on the relative live birth rates after in vitro maturation compared with in vitro fertilisation.

- Data are being collected from a single study centre, limiting the generalisability of the results.

- The study population is from Vietnam, meaning that the results may not apply to patients of different ethnicities, particularly given the different polycystic ovary syndrome phenotype in Asian patients.

hyperstimulation $(\mathrm{COH})$ and in vitro fertilisation (IVF). However, COH is costly and is associated with a high risk of ovarian hyperstimulation syndrome (OHSS) in women with PCOS. ${ }^{5}{ }^{6}$ In addition, IVF may be less successful in women with PCOS due to the retrieval of immature or poor quality oocytes, leading to reduced fertilisation, cleavage, pregnancy and live birth rates, and a higher rate of miscarriage. ${ }^{7}$

In vitro maturation (IVM) is an alternative to conventional IVF and was first used to achieve successful pregnancy and childbirth in $1991 .{ }^{8}$ In IVM, follicle-stimulating hormone treatment is not used at all or only used for 3 days as ovarian priming. Immature oocytes (germinal vesicle stage) are harvested from follicles in the early antral stage $(2-10 \mathrm{~mm})$, then incubated in IVM medium for 30 hours to mature in vitro until metaphase II. Matured oocytes can then be used for standard IVF or intracytoplastmic sperm injection (ICSI) procedures, although the latter is generally preferred to avoid zona hardening of the oocytes. 
Successful fertilisation, embryo development and term pregnancy resulting from IVM oocytes have been reported in women with PCOS. ${ }^{9}$ Although there has been some concerns about the effectiveness of IVM and the potential for genetic abnormalities in the resulting infants, ${ }^{10-13}$ there is now growing evidence that pregnancy outcomes after IVM are similar to those after conventional IVF. ${ }^{14}$ However, a Cochrane review published in 2013 concluded that there was no evidence from randomised controlled trials on which to base recommendations regarding the use of IVM before IVF or ICSI for women with PCOS in clinical practice. ${ }^{9}$ Therefore, this study was designed to investigate the effectiveness and safety of one cycle of IVM (without human chorionic gonadotropin (hCG) priming) versus one cycle of $\mathrm{COH} / \mathrm{IVF}$ with gonadotropin-releasing hormone $(\mathrm{GnRH})$ agonist triggering (IVF with segmentation) in women with PCOS or a high antral follicle count (AFC).

\section{METHODS AND ANALYSIS}

\section{Study design and ethical considerations}

The study has a randomised, controlled design and will be conducted at My Duc Hospital, Ho Chi Minh City, Vietnam, according to the principles outlined in the Declaration of Helsinki and its amendments, in accordance with the Medical Research Involving Human Subjects Act (WMO) and using Good Clinical Practice. Potentially eligible women will be given information about the study during their first consultation (at least 2 weeks before the start of their menstrual cycle). Screening for eligibility will be performed by treating physicians on day 2 of the menstrual cycle, and patients will be given a copy of the informed consent documents. Written informed consent will be obtained by the investigator from all women prior to enrolment. Women will be randomised (1:1) to IVM or IVF using block randomisation by an independent study coordinator via telephone, using a computer-generated random list (block size 2, 4 or 8 ). The study is currently recruiting, with an estimated duration of 24 months (recruitment completed by 31 December 2019).

Participants can leave the study at any time for any reason if they wish to do so without any consequences for their treatment. The investigator can decide to withdraw a subject from the study for urgent medical reasons. After randomisation, if a participant wishes to change her assigned protocol, she will be considered as a crossover subject. Subjects who choose to transfer more than two embryos after randomisation will be considered as protocol deviations.

\section{Patient and public involvement}

Patients and/or public were not involved in the study design and study enrolment.

\section{Participants}

Eligible women are those with high AFC, including PCOS (hyperandrogenism or ovulation disorder plus polycystic ovaries on ultrasound based on the 2003 Rotterdam criteria $)^{15}$ or a high AFC ( $\geq 24$ antral follicles in both ovaries). In addition, women must have an indication for ART, are permanent residents in Vietnam, have undergone $\leq 2$ previous IVM/IVF attempts, agree to have all embryos frozen on day 3 and $\leq 2$ embryos transferred in a subsequent frozen transfer and are not participating in another IVF study at the same time. Oocyte donation and preimplantation genetic diagnosis cycles are excluded.

\section{Treatment groups}

In vitro maturation

Women with a normal cycle length ( $\leq 35$ days) will receive injected highly purified human menopausal gonadotropin (hp-hMG; Menopur, Ferring) $150 \mathrm{IU} /$ day starting on day 2 or 3 of the spontaneous menstrual cycle. Oocyte retrieval will be performed 42 hours after the last hp-hMG injection. Women who do not have a normal cycle length (>35 days; 4-9 menstrual cycles in a year or amenorrhoea) will take an oral contraceptive for 2 weeks, then receive hp-hMG 150 IU/day (hp-hMG; Menopur, Ferring) injection for 2 days starting 5 days later.

In all participants, ultrasound will be performed on the second day of gonadotrophin injection, and Oocyte pick-up (OPU) is scheduled for 42 hours after the last gonadotrophin injection. After oocyte pick-up, all oocytes will be placed in prematuration medium (CAPA Pre-maturation in Medicult IVM medium, Origio, Denmark) for 24 hours, then transferred to maturation culture (Medicult IVM system with phenol red, Origio, Denmark) for 30 hours.

\section{IVF with segmentation}

All women in this group will undergo $\mathrm{COH}$ using a hp-hMG/GnRH antagonist protocol, with an HP-hMG dose of 150-225 IU/day (Menopur, Ferring), depending on age and body mass index. Follicular development will be monitored using ultrasound scanning, and estradiol and progesterone levels. When at least two leading follicles reach $17 \mathrm{~mm}$ in diameter, GnRH agonist (GnRHa) triggering with triptorelin $0.2 \mathrm{mg}$ (Diphereline, Ipsen Beaufour) will be administered, and oocyte retrieval performed 36 hours later.

\section{Fertilisation and embryo assessment}

In both groups, insemination of mature oocytes will be performed using ICSI at 3-4hours after oocyte retrieval or maturation check. Fertilisation check will be performed under an inverted microscope at 16-18 hours after insemination. Embryo evaluation will be performed at $68 \pm 1$ hours after fertilisation using the Istanbul consensus. ${ }^{16}$

\section{Freeze-all and frozen embryo transfer}

All embryos will be frozen on day 3. Frozen transfer of a maximum of 2 embryos will be performed in a subsequent cycle. The endometrium will be prepared using oral estradiol valerate (Valiera; Laboratories Recalcine) $8 \mathrm{mg} /$ day starting from the second or third day of the menstrual 
cycle. Endometrial thickness will be monitored from day 6 onwards, and vaginal progesterone (Cyclogest; Actavis) $800 \mathrm{mg} /$ day will be started when endometrial thickness reaches $8 \mathrm{~mm}$ or more. A maximum of two embryos will be thawed on the day of embryo transfer, 3 days after the start of progesterone. Two hours after thawing, surviving embryos will be transferred into the uterus under ultrasound guidance. Clinicians performing frozen embryo transfer were unaware of group allocation throughout this process. Transfer of one or two embryos was based on patient preference. When women have more than two embryos frozen, the procedure will be repeated in subsequent cycles if first transfer is unsuccessful.

\section{Outcomes}

\section{Primary outcome}

The primary endpoint is live birth after the first embryo transfer of the started treatment cycle. Cycles in which no embryo is available for transfer will be considered as failures. Live birth is defined as the birth of at least one newborn after 24 weeks' gestation that exhibits any sign of life (twins will be a single count). To allow assessment of the timing of live birth, the rate of ongoing pregnancy at 12 weeks will be used in calculations, conditional on the fact that this ongoing pregnancy results in live birth.

\section{Secondary outcomes}

A number of fertility, maternal safety, pregnancy complication, obstetric and perinatal complication, and neonatal complication outcomes were assessed. Full details and definitions are provided in table 1.

\section{Safety}

The primary investigator will inform subjects and the reviewing accredited medical research ethics committee if anything occurs that would suggest that the disadvantages of participation may be significantly greater than was foreseen in the research proposal. The study will be suspended pending further review by the accredited medical research ethics committee, except where suspension would jeopardise the participants' health. The investigator will ensure that all subjects are kept informed.

Adverse events are defined as any undesirable experience occurring to a subject during a clinical trial, whether or not considered related to the intervention. All adverse events reported spontaneously by the subject or observed by the investigator or their staff will be recorded. Routine assessments for OHSS were performed on day 3 post oocyte retrieval in both groups. At other times, OHSS was evaluated if symptoms were reported by the patient. OHSS was classified using the flow diagram developed by Humaidan and colleagues for use in clinical trial settings. ${ }^{17}$

A serious adverse event (SAE) is defined as any untoward medical occurrence or effect that results in death, is life-threatening (at the time of the event), requires hospitalisation or prolongation of an existing hospitalisation, results in persistent or significant disability or incapacity, is a congenital anomaly or birth defect, is a new event of the trial likely to affect the safety of the subjects such as an unexpected outcome of an adverse reaction. All SAEs will be reported to the accredited ethics committee that approved the protocol, according to the requirements of that committee.

All adverse events will be followed until they have abated or until a stable situation has been reached. Depending on the event, follow-up may require additional tests or medical procedures as indicated, and/or referral to a general physician or medical specialist.

\section{Cost-effectiveness}

Cost data will be collected for a supplementary analysis and will be reported in a separate paper. At the time of pregnancy testing, participants will be questioned to determine the indirect costs of treatment (see online appendix). For participants with treatment-related complications, the cost of managing these complications will be determined on patient discharge. Costs per patient will be calculated and grouped into categories. Direct costs include those for examinations, drugs, freezing, thawing, complications, pregnancy and delivery. Indirect costs include travel, absence from work and accommodation (see online appendix).

\section{Genetic and epigenetic analysis of newborns}

The genetics and epigenetics of babies born from IVM and IVF will be investigated as a supplementary analysis and reported separately. Prior to initiation of IVF or IVM, $2.5 \mathrm{~mL}$ of maternal whole blood will be collected and stored in PAXgene blood DNA tubes (Qiagen) at $-80^{\circ} \mathrm{C}$. This will serve as a control for the genomic variants between mother and fetus. At the time of delivery, materials including cord blood, neonatal buccal smear and placental tissue will be collected to study DNA methylation patterns and gene expression. A total of $11 \mathrm{~mL}$ of cord blood will be collected, of which $8.5 \mathrm{~mL}$ will be transferred to PAX gene blood DNA tubes and $2.5 \mathrm{~mL}$ to PAXgene blood RNA tubes (Qiagen), all of which will be stored at $-80^{\circ} \mathrm{C}$. A buccal smear will be collected by placing a sponge into the neonatal cheek pouch immediately after delivery, then the sponge will be gently moved along the gums and inner cheeks for 30 s to soak up as much saliva as possible. Saliva samples will be stored at room temperature. The placenta will be weighed before collection of placental tissues. Four full thickness vertical samples (encompassing both maternal and foetal sides of the placenta) will be cut from areas without large blood vessels or fibrous tissue. Each piece (maximum size $4 \times 10 \times 10 \mathrm{~mm}$ ) will be rinsed in sterile phosphate buffered saline (PBS) 1X until all the blood has been removed. The tissue is then dabbed in sterile gauze until dry, placed into a standard tissue cassette and then transferred into PAXgene tissue containers (Qiagen). All tissue samples should be stored in the fixative solution in the container within $30 \mathrm{~min}$ after the start of tissue collection. All tissue containers will be stored at $-80^{\circ} \mathrm{C}$. Next-generation 
Table 1 Secondary endpoints and their definition

\section{Secondary endpoint \\ Definition}

Fertility outcomes

Positive pregnancy test

Serum $\mathrm{hCG}>5 \mathrm{mlU} / \mathrm{mL}$ after the completion of the first transfer

Clinical pregnancy

$\geq 1$ gestational sac on ultrasound at 7 weeks' gestation with the detection of heart beat activity after the completion of the first transfer

Ongoing pregnancy

Pregnancy with detectable heart rate at $\geq 12$ weeks' gestation after the completion of the first transfer

Number of top-quality embryos Based on Istanbul consensus criteria ${ }^{16}$

Number of freezable embryos

Number of frozen embryos after completion of the first transfer

Time to ongoing pregnancy

Time from randomisation to detection of ongoing pregnancy after completion of the first transfer

Cumulative ongoing pregnancy rate

Ongoing pregnancy rate at 6 and 12 months after randomisation

Maternal safety

OHSS

Classified as moderate or severe using a flow diagram developed for use in clinical trials $^{17}$

Pregnancy complications*

Ectopic pregnancy

Implantation outside the uterine cavity

Miscarriage

Multiple pregnancy

Pregnancy loss at $<12$ weeks

Multiple delivery

$\geq 1$ gestational sac at early pregnancy ultrasound (6-8 weeks' gestation)

Birth of $\geq 1$ baby beyond 24 weeks' gestation

Obstetric and perinatal complications ${ }^{\star}$

Gestational diabetes mellitus

Development of diabetes during pregnancy

Hypertensive disorders of pregnancy

Antepartum haemorrhage

Pregnancy-induced hypertension, pre-eclampsia and eclampsia ${ }^{18}$

Preterm delivery

Including placenta previa, placenta accreta and unexplained

Spontaneous preterm birth

Delivery at $<24,<28,<32,<37$ completed weeks

latrogenic preterm birth

Delivery at $<24,<28,<32,<37$ completed weeks

Birth weight

Delivery at $<24,<28,<32,<37$ completed weeks

Low birth weight

In grams; of singletons and twins

Very low birth weight

$<2500 \mathrm{~g}$ at birth

High birth rate

$<1500 \mathrm{~g}$ at birth

Very high birth rate

$>4000 \mathrm{~g}$ at birth

Large for gestational age

Small for gestational age

$>4500 \mathrm{~g}$ at birth

Neonatal complications ${ }^{\star}$

Congenital anomaly

Admission to NICU

Birth weight $>90$ th centile for gestation, based on standardised ethnicity-based charts Birth weight $<10$ th centile for gestation, based on standardised ethnicity-based charts

*All assessed after completion of the first transfer, at 6 months after randomisation and at 12 months after randomisation.

hCG, human chorionic gonadotropin; NICU, neonatal intensive care unit; OHSS, ovarian hyperstimulation syndrome.

sequencing will be used to analyse genetic and epigenetic variants.

\section{Sample size calculation}

The current live birth rate for women with PCOS at the study centre is $45 \%$ per initiated cycle. Assuming the difference between IVM and IVF is $5 \%$, to achieve a study power of $90 \%$ with a one-sided alpha of 0.025 , a non-inferiority margin of $-10 \%$ and a loss to follow-up or protocol deviation rate of $15 \%$, the goal is to randomise 546 participants (273 per arm). To maximise retention in the trial, consultation was made available to patients to ensure they understood the procedures well and to address any questions or complaints that arose during the study.

\section{Confidentiality and ownership of trial data}

Lan N Vuong, Toan D Pham and Ben W Mol will have access to the final trial dataset. Any confidential information relating to the trial, including any data and results from the trial will be the exclusive property of My Duc 
Hospital. The investigator and any other persons involved in the trial will protect the confidentiality of this proprietary information belonging to My Duc Hospital.

\section{Statistical analysis}

All analyses will be conducted on an intention-to-treat basis using the R statistical program (R V.3.5.0; 2018 The R Foundation for Statistical Computing). Per-protocol analyses may be conducted but these would be considered exploratory only. If a subject withdraws consent, no further data will be analysed, but existing samples can be included in the analysis (unless the patient requests destruction of existing samples). Baseline data will be presented using descriptive statistics (mean and SD for normally distributed variables, or median and IQR for skewed variables). Categorical data will be presented as number $(\%)$.

The rate of live birth and the associated $95 \%$ CI will be estimated for each group and compared between groups using the exact method for binomial proportion. The study is designed as a non-inferiority study in which the maximal acceptable difference in live birth rate between IVM and IVF will be 5\%, margin of $10 \%$. Kaplan-Meier survival curves for the cumulative live birth rates in each treatment group will be constructed. The log rank test and Cox regression model will be used to assess betweengroup differences in the cumulative pregnancy rates. Differences between groups in secondary outcome variables will be analysed using Student's t-test or Wilcoxon signed-rank test for normally distributed or skewed variables, and Fisher's exact test for categorical variables, and reported as relative risk with $95 \%$ CI.

\section{Interim analysis and monitoring}

Interim analysis will be performed after enrolment of the first 273 participants. An independent Data Safety Monitoring Committee (DSMC) will be convened (members: JLH Evers, S Bhattacharya, E Schuit; copy of full charter provided in the online appendix). The DSMC will assess the ongoing pregnancy because data on live birth will not be available. The DSMC will also review any SAEs that have occurred. The interim analysis will be conducted using a two-sided significant test with the Haybittle-Peto spending function and a type I error rate of $5 \%$ with stopping criteria of $\mathrm{p}<0.001$ ( $\mathrm{Z}$ alpha $=3.29)$.

Independent study monitoring will be performed monthly by a clinical research associate from the My Duc Hospital Clinical Research Department to ensure adherence to the protocol, International Conference on Harmonisation-Good Clinical Practice, standard operating procedures and applicable regulatory requirements, maintenance of trial-related source records, completeness, accuracy and verifiability of case report form entries compared with source data.

\section{Data handling}

Data will be collected using a questionnaire and recorded using RedCap software (Vanderbilt University). All data are entered into the database twice. The first data entry is made within a day after embryo transfer. The second is undertaken at the termination of the study. Data from the two entries will be used to check for potential inconsistencies, and any inconsistencies will be adjudicated using the original patient medical record. Data monitoring will be done by the principal investigator. Patient privacy will be ensured by allocation of a five-digit number to each participant, which will be used on all study documentation, with the participant code only available to the local investigator.

\section{Missing data}

Missing observations for the primary endpoint (ongoing pregnancy rate) will be imputed as 'negative' irrespective of the reason why data are not recorded. Missing observations for the supportive secondary endpoints (positive beta-hCG rate, clinical pregnancy rate and vital pregnancy rate) will be imputed as 'negative' unless a positive result is observed at a later pregnancy assessment. For example, if the outcome of beta-hCG is missing but clinical pregnancy is positive then beta-hCG will be imputed as 'positive'. For subjects with embryo transfer but missing information on the number of viable fetuses at 10-11 weeks after transfer, the number of viable fetuses will be imputed as zero irrespective of why data are not recorded. For subjects with transfer but missing information on the number of gestational sacs 5-6 weeks after transfer, the number of gestational sacs will be imputed as the number of viable fetuses at 10-11 weeks after transfer. For adverse events, missing values will be treated as missing, except for causality, intensity and seriousness of adverse events, where a worst-case approach will be used. Missing values will not be imputed for any of the other secondary endpoints.

\section{End of study}

The principal investigator will notify the accredited ethical committee and the competent authority of the end of the study within a period of 90 days. The end of the study is defined as the last participant's last visit. In the event of early study termination, the principal investigator will notify the accredited ethical committee within 15 days, including the reasons for premature termination.

\section{Ethics and dissemination}

Any change to the study protocol will be documented in a protocol amendment. Amendments are submitted for consideration to the approving ethics committee. If new information becomes available that may be relevant to a subject's willingness to continue participation in the trial, a new subject information and informed consent form will be forwarded to the ethics committee. The trial subjects will be informed about this new information and consent will be obtained again. Changes will be updated on trial registration website clinicaltrials.gov. Changes to the protocol to eliminate immediate hazard(s) to trial subjects may be implemented prior to ethics committee approval or receipt of a favourable opinion. 
No specific arrangements will be made between any sponsors and the investigator concerning the public disclosure and publication of the research data. The principal investigator will prepare a manuscript detailing the results of the main study as soon as appropriate and submit this to a peer-reviewed medical journal. Supplementary analyses will be analysed and reported separately.

\section{Author affiliations}

${ }^{1}$ Department of Obstetrics and Gynaecology, University of Medicine and Pharmacy at Ho Chi Minh City, Ho Chi Minh City, Vietnam

${ }^{2}$ IVFMD, My Duc Hospital, Ho Chi Minh City, Vietnam

${ }^{3}$ Robinson Research Institute and Adelaide Medical School, The University of

Adelaide, Adelaide, South Australia, Australia

${ }^{4}$ Fertility SA, Adelaide, South Australia, Australia

${ }^{5}$ Follicle Biology Laboratory, Free University of Brussels (VUB), Brussels, Belgium

${ }^{6}$ School of Women's and Children's Health, University of New South Wales, Sydney, New South Wales, Australia

${ }^{7}$ Department of Obstetrics and Gynaecology, School of Medicine, Monash University, Melbourne, Victoria, Australia

Contributors Study concept and design: LNV, TMH, VQD, RW, RJN, JS, RBG, BWM. Acquisition of data: LNV, VNAH, TMH, VQD, THP, NHG, AHL, TDP. Analysis and interpretation of data: LNV, TDP, VNAH, TMH, RW, RJN, JS, RBG, BWM. Drafting of the manuscript: LNV and BWM. Critical revision of the manuscript for important intellectual content: LNV, VNAH, TMH, VQD, THP, NHG, AHL, TDP, RW, RJN, JS, RBG, BWM. Statistical analysis: TDP and LNV. Study supervision: RJN and BWM.

Funding This work was supported by Ferring grant number 000323 and sponsored by My Duc Hospital.

Disclaimer My Duc Hospital hosted meetings of the scientific committee and ethical review board. Otherwise, neither the sponsor nor funder had any role in the study design, collection, management, analysis and interpretation of data, writing of the report or the decision to submit for publication.

Competing interests LNV has received speaker and conference fees from Merck, grant, speaker and conference fees from Merck Sharpe and Dohme, and speaker, conference and scientific board fees from Ferring. TMH has received speaker fees from Merck, Merck Sharp and Dohme, and Ferring. RJN has received grants and conference fees from Merck, grants and speaker fees from Merck Sharp and Dohme, and conference and scientific board fees for Ferring. BWM has acted as a paid consultant to Merck, ObsEva and Guerbet, and is the recipient of grant money from the NHMRC Practitioner Fellowship. RBG reports grants from National Health and Medical Research Council of Australia. JS reports grants from Fund for research Flanders, and has a patent W0 201609497 A1 pending. TDP, VQD, VNAH, NHG, AHL, THP and RW have no conflicts of interest to declare.

Patient consent for publication Obtained.

Ethics approval Ethic Review Board My Duc Hospital.

Provenance and peer review Not commissioned; externally peer reviewed.

Open access This is an open access article distributed in accordance with the Creative Commons Attribution Non Commercial (CC BY-NC 4.0) license, which permits others to distribute, remix, adapt, build upon this work non-commercially, and license their derivative works on different terms, provided the original work is properly cited, appropriate credit is given, any changes made indicated, and the use is non-commercial. See: http://creativecommons.org/licenses/by-nc/4.0/.

\section{REFERENCES}

1. Ding T, Hardiman PJ, Petersen I, et al. The prevalence of polycystic ovary syndrome in reproductive-aged women of different ethnicity: a systematic review and meta-analysis. Oncotarget 2017;8:96351-8.

2. Azziz R, Marin C, Hoq L, et al. Health care-related economic burden of the polycystic ovary syndrome during the reproductive life span. $J$ Clin Endocrinol Metab 2005;90:4650-8.

3. ESHRE Capri Workshop Group. Health and fertility in World Health Organization group 2 anovulatory women. Hum Reprod Update 2012;18:586-99.

4. Thessaloniki ESHRE/ASRM-Sponsored PCOS Consensus Workshop Group. Consensus on infertility treatment related to polycystic ovary syndrome. Hum Reprod 2008;23:462-77.

5. Brinsden PR, Wada I, Tan SL, et al. Diagnosis, prevention and management of ovarian hyperstimulation syndrome. Br J Obstet Gynaecol 1995;102:767-72.

6. MacDougall MJ, Tan SL, Jacobs HS. In-vitro fertilization and the ovarian hyperstimulation syndrome. Hum Reprod 1992;7:597-600.

7. Qiao J, Feng HL. Extra- and intra-ovarian factors in polycystic ovary syndrome: impact on oocyte maturation and embryo developmental competence. Hum Reprod Update 2011:17:17-33.

8. Cha KY, Koo JJ, Ko JJ, et al. Pregnancy after in vitro fertilization of human follicular oocytes collected from nonstimulated cycles, their culture in vitro and their transfer in a donor oocyte program. Fertil Steril 1991;55:109-13.

9. Siristatidis CS, Vrachnis N, Creatsa M, et al. In vitro maturation in subfertile women with polycystic ovarian syndrome undergoing assisted reproduction. Cochrane Database Syst Rev 2013;10:CD006606.

10. Kerjean A, Couvert P, Heams $T$, et al. In vitro follicular growth affects oocyte imprinting establishment in mice. Eur J Hum Genet 2003;11:493-6.

11. Nogueira D, Staessen $\mathrm{C}$, Van de Velde $\mathrm{H}$, et al. Nuclear status and cytogenetics of embryos derived from in vitro-matured oocytes. Fertil Steril 2000;74:295-8.

12. Young LE, Fernandes K, McEvoy TG, et al. Epigenetic change in IGF2R is associated with fetal overgrowth after sheep embryo culture. Nat Genet 2001;27:153-4.

13. Zhang XY, Ata B, Son WY, et al. Chromosome abnormality rates in human embryos obtained from in-vitro maturation and IVF treatment cycles. Reprod Biomed Online 2010;21:552-9.

14. Walls ML, Hunter T, Ryan JP, et al. In vitro maturation as an alternative to standard in vitro fertilization for patients diagnosed with polycystic ovaries: a comparative analysis of fresh, frozen and cumulative cycle outcomes. Hum Reprod 2015;30:88-96.

15. Rotterdam ESHRE/ASRM-Sponsored PCOS consensus workshop group. Revised 2003 consensus on diagnostic criteria and longterm health risks related to polycystic ovary syndrome (PCOS). Hum Reprod 2004;19:41-7.

16. ALPHA Scientists In Reproductive MedicineESHRE Special Interest Group Embryology. Istanbul consensus workshop on embryo assessment: proceedings of an expert meeting. Reprod Biomed Online 2011;22:632-46.

17. Humaidan P, Nelson SM, Devroey P, et al. Ovarian hyperstimulation syndrome: review and new classification criteria for reporting in clinical trials. Hum Reprod 2016;31:1997-2004.

18. Queensland Clinical Guidelines. Hypertensive disorders of pregnancy (2015). 2015. https://http://www.health.qld.gov.au/qcg/documents/ g-hdp.pdf (Accessed 3 Oct 2016). 